\title{
Mathematical model of Malaria with flooding in Thailand
}

\author{
Puntani Pongsumpun \\ Department of Mathematics, Faculty of Science, \\ King Mongkut's institute of Technology Ladkrabang, \\ Chalongkrung road, Ladkrabang, Bangkok 10520, Thailand
}

\begin{abstract}
Malaria is occurred by biting of infected Anopheles mosquitoes. Four species of Plasmodium are Plasmodium falciparum, Plasmodium vivax, Plasmodium ovale and Plasmodium malariae. This disease is found in tropical countries, especially Thailand. We consider the effect of flooding in Thailand with the transmission of Malaria disease. Mathematical model is formulated by using theory of modeling. The variables and parameters are defined corresponding to the characteristic of this disease. Our model is analyzed by using dynamical modeling method. Numerical simulations are shown comparing with the analytical solutions.
\end{abstract}

Keywords: Anopheles mosquito, Malaria disease, Mathematical model, Plasmodium

\section{Introduction}

Anopheles mosquitoes is the epidemic vector of this disease. This disease is caused by the multiplication of protozoa parasite of the genus Plasmodium. P.falciparum, P.vivax, P.malariae and P.ovale are four species of Plasmodiums. Greater than three hundred million Malaria cases are reported per year [1]. Each species are discovered in the different areas. Plasmodium falciparum is found on the tropic and subtropics such as Africa, South America and Asia. Plasmodium vivax is found in the widest area. It can be found in many temperate zones, subtropics and tropic such as China, Turkey, Latin America and Asia. Plasmodium malariae is found in the same area as Plasmodium falciparum but is much less common in areas such as Central America. Plasmodium ovale is found predominantly in tropic Africa, but many occur in the West Pacific. Blood transfusion can receive this disease accidentally. This is one of the reasons why people who have been infected with disease can never donate blood. Infection of a newborn from an infected mother also happens, but it is comparatively rare [2]. In the first two months of life, children may not contact malaria or their manifestations may be mild with low-grade parasitemia[2], Host, agent and environment are three factors which influence to the transmission of Malaria. The most important environmental factors are temperature and humidity. When the temperature is under 16, Malaria parasites stop developing in the mosquitoes. The best temperature for the development of this disease is between $20-30 \mathrm{C}$. The average relative humidity is at least $60 \%$ [3]. There are 4 cycles of this mosquito: egg, larva, pupa and adult. The male Anopheles feeds on nectar and fruit juices while the female takes both these plant products and blood [4]. The female may lay several batches of eggs during her lifetime. The eggs hatch within $2-3$ days, releasing the larvae into water, the larvae transform into the nonfeeding pupae. Within the pupae, over a period of $2-4$ days, metamorphosis takes place, terminating in the materialization of the adults [5]. Sick due to this disease causes significant economic loss. Situations of global temperatures increase the life cycle of a mosquito vector[3]-[5]. The period of mosquito eggs and larvae molt growth as a mosquito can take 7-10 days. The average life expectancy of the mosquito is 45 days. In Thailand, Malaria is found along the border with Burma, Cambodia, and Malaysia. The original Malaria model is usually described by the RossMacDonald (RM) model [6].In 2001, Kammanee A et al.[7] formulated the mathematical model for Malaria transmission and basic reproductive number is found to reduce the outbreak of the disease. To describe the flooding in Thailand with the transmission of Malaria, we incorporate the flooding parameters to our model. Analytical result and numerical simulations are used in our study to suggest the way for reducing the disease outbreak.

\section{Mathematical Model}

We study the transmission of Malaria with flooding in Thailand by formulating the differential equations. We use the knowledge of mathematical model to describe the transmission of this disease. We consider the 
dynamical equations of human and mosquitoes. The differential equations for describing the transmission of this disease is

$$
\begin{aligned}
S_{n}^{\prime}(t) & =h N_{n}-\gamma_{n} S_{n} I_{v}-d S_{n} \\
E_{n}^{\prime}(t) & =\gamma_{n} S_{n} I_{v}-\frac{1}{I I_{n}} E_{n}-d E_{n} \\
I_{n}^{\prime}(t) & =\frac{1}{I I_{n}} E_{n}-(\gamma+d) I_{n} \\
R_{n}^{\prime}(t) & =\gamma I_{n}-d R_{n} \\
S_{v_{f}}^{\prime}(t) & =A_{f}-\gamma_{v_{f}} S_{v_{f}} I_{n}-d_{v} S_{v_{f}} \\
I_{v_{f}}^{\prime}(t) & =\gamma_{v_{f}} S_{v_{f}} I_{n}-d_{v} I_{v_{f}} \\
S_{v_{n}}^{\prime}(t) & =A_{n_{f}}-\gamma_{v_{n}} S_{v_{n_{f}}} I_{n}-d_{v} S_{v_{n}} \\
I_{v_{n}}^{\prime}(t) & =\gamma_{v_{n}} S_{v_{n_{f}}} I_{n}-d_{v} I_{v_{n}}
\end{aligned}
$$

where $N_{n}=S_{n}+E_{n}+I_{n}+R_{n}, N_{v_{f}}=S_{v_{f}}+I_{v_{f}}$ and $N_{v_{n f}}=S_{v_{n f}}+I_{v_{n f}}$.

The variables and parameters in our equations are described as follows:

$S_{n}(t)$ is the size of susceptible human population at time $t$,

$E_{n}(t)$ is the size of exposed human population at time t,

$I_{n}(t)$ is the size of infectious human population at time $\mathrm{t}$,

$R_{n}(t)$ is the size of recovered human population at time $\mathrm{t}$,

$S_{v_{f}}(t)$ is the size of susceptible mosquito during the flood at time t,

$I_{v_{f}}(t)$ is the size of infectious mosquito during the flood at time $\mathrm{t}$,

$S_{v_{n}}(t)$ is the size of susceptible mosquito during the non-flood at time t,

$I_{v_{f}}(t)$ is the size of infectious mosquito during the non-flood at time t,

$\mathrm{h}$ is the birth rate of human population,

$\mathrm{d}$ is the death rate of human population,

$\gamma_{n}$ is the transmission rate of Plasmodium malaria from mosquito to human,

$I I_{n}$ is the incubation period of Plasmodium malaria in human,

$N_{n}$ is the size of human population,

$N_{v f}$ is the size of mosquitoes during flooding time,

$N_{v_{n}}$ is the size of mosquitoes during non-flooding time,

$\gamma$ is the recovery rate of human population,

$A_{f}$ is the constant recruitment rate of mosquitoes during flooding time,

$A_{n f}$ is the constant recruitment rate of mosquitoes during non-flooding time,

$\gamma_{v_{f}}$ is the transmission rate of Plasmodium malaria from human to mosquitoes during flooding time,

$\gamma_{v_{n}}$ is the transmission rate of Plasmodium malaria from human to mosquitoes during non-flooding time, $d_{v}$ is the death rate of mosquitoes,

Suppose that the size of human and mosquitoes are constant, then

$N_{n}^{\prime}=S_{n}^{\prime}+E_{n}^{\prime}+I_{n}^{\prime}+R_{n}^{\prime}=0, N_{v_{f}}^{\prime}=S_{v_{f}}^{\prime}+I_{v_{f}}^{\prime}=0$ and $N_{v_{n f}}^{\prime}=S_{v_{n f}}^{\prime}+I_{v_{n f}}^{\prime}=0$. From the above equations, we get h=d, $N_{v_{f}}=A_{f} / d_{v}$ and $N_{v_{n f}}=A_{n f} / d_{v}$.

Normalizing the above equations by letting $s_{n}=S_{n} / N_{n}, e_{n}=E_{n} / N_{n}, i_{n}=I_{n} / N_{n}, r_{n}=R_{n} / N_{n}$,

$$
\begin{aligned}
& s_{v_{f}}=S_{v_{f}} / N_{v}, i_{v_{f}}=I_{v_{f}} / N_{v}, s_{v_{f}}=S_{v_{f}} / N_{v_{f}}, i_{v_{f}}=I_{v_{f}} / N_{v_{f}}, \\
& s_{v_{n_{f}}}=S_{v_{n_{f}}} / N_{v_{n_{f}}}, i_{v_{n_{f}}}=I_{v_{n_{f}}} / N_{v_{n_{f}}} .
\end{aligned}
$$


Thus, the reduced equations become:

$$
\begin{aligned}
& s_{n}^{\prime}(t)=h-\left(d+\gamma_{n} i_{v}\right) s_{h} \\
& e_{n}^{\prime}(t)=\gamma_{n} s_{n} i_{v}-\frac{1}{I I_{n}} e_{n}-d e_{n} \\
& i_{n}^{\prime}(t)=\frac{1}{I I_{n}} e_{n}-(\gamma+d) i_{n} \\
& i_{v_{f}}^{\prime}(t)=\gamma_{v_{f}} s_{v_{f}} i_{n} N_{n}-d_{v} i_{v_{f}} \\
& i_{v_{n f}^{\prime}}^{\prime}(t)=\gamma_{v_{n}} s_{v_{n}} i_{n} N_{n}-d_{v} i_{v_{n}}
\end{aligned}
$$

\section{Analysis of Mathematical Model}

\subsection{Analytical Solutions}

The standard dynamical method is used for analysis our model. Steady states of our equations are found by setting (9)-(13) to zero, then we obtain the steady states:

i) Disease free steady state: $(1,0,0,0,0)$ and

ii) Endemic steady state: $\left(s_{n}, e_{n}, i_{n}, i_{v_{f}}, i_{v_{n f}}\right)$ where

$$
\begin{aligned}
& s_{n}^{*}=\frac{h}{h+\gamma_{n}\left(i_{v_{f}}^{*}+i_{v_{n}}^{*}\right)}, e_{n}^{*}=\frac{\gamma_{n} I I_{n} i_{v^{\prime}}^{*} s_{h}^{*}}{1+d I I_{n}}, \\
& i_{v_{f}}^{*}=\frac{\gamma_{v_{f}} \mathrm{~N}_{\mathrm{h}}}{(h+\gamma) I I_{n}\left(d_{v}+\gamma_{v_{f}} i_{n}^{*} \mathrm{~N}_{\mathrm{h}}\right)} e_{n}^{*} \text { and } i_{v_{n f}}^{*}=\frac{\gamma_{v_{f}} \mathrm{~N}_{\mathrm{h}}}{(h+\gamma) I I_{n} d_{v}+e_{n}^{*} \gamma_{v_{n}} \mathrm{~N}_{\mathrm{h}}} e_{n}^{*}
\end{aligned}
$$

where

$$
\begin{aligned}
& i_{n}^{*}=\frac{1}{2(d+\gamma)\left(d+2 \gamma_{n}\right) \gamma_{v_{f}} \gamma_{v_{n}}\left(1+d \mathrm{II}_{\mathrm{n}}\right) \mathrm{N}_{\mathrm{h}}^{2}}\left(-d_{v}(d+\gamma)\left(d+\gamma_{n}\right)\left(\gamma_{v_{f}}+\gamma_{v_{n}}\right)\left(1+d \mathrm{II}_{\mathrm{n}}\right) \mathrm{N}_{\mathrm{h}}\right. \\
& +2 \gamma_{n} \gamma_{v_{f}} \gamma_{v_{n}} \mathrm{~N}_{\mathrm{h}}^{2}+\sqrt{\begin{array}{l}
\left(\mathrm { N } _ { \mathrm { h } } ^ { 2 } \left(\left(d_{v}(d+\gamma)\left(d+\gamma_{n}\right)\left(\gamma_{v_{f}}+\gamma_{v_{n}}\right)\left(1+d \mathrm{II}_{\mathrm{n}}\right)-2 \gamma_{n} \gamma_{v_{f}} \gamma_{v_{n}} \mathrm{~N}_{\mathrm{h}}\right)^{2}\right.\right. \\
-4 d_{v}(d+\gamma)\left(d+2 \gamma_{n}\right) \gamma_{v_{f}} \gamma_{v_{n f}}\left(1+d \mathrm{II}_{\mathrm{n}}\right)\left(d d_{v}(d+\gamma)\left(1+d \mathrm{II}_{\mathrm{n}}\right)\right. \\
\left.\left.\left.\left.-\gamma_{n}\left(\gamma_{v_{f}}+\gamma_{v_{n f}}\right) \mathrm{hN}_{\mathrm{h}}\right)\right)\right)\right)
\end{array}}
\end{aligned}
$$

By using Standard dynamical modeling method, the local stability of each steady state is determined by considering the signs of eigenvalues. If the signs of all eigenvalues give negative, then we can conclude that that steady state is local stability [8-10]. The characteristic equation is defined by following equation:

$$
|\mathrm{J}-\kappa \mathrm{I}|=0
$$

where $|\mathrm{A}|$ means determinant of $\mathrm{A}, \mathrm{J}$ is the Jacobian matrix, $\kappa$ is the eigenvalues and $\mathrm{I}$ is the identity matrix . After evaluating our model, the condition for negative real parts of eigenvalues is $R_{0}>1$, where

$$
R_{0}=\frac{\gamma_{n} N_{n}\left(\gamma_{v_{f}}+\gamma_{v_{n_{f}}}\right)}{d_{v}(d+\gamma)\left(1+d \mathrm{II}_{\mathrm{n}}\right)} \text {. }
$$


Therefore, we can conclude that the disease free steady state is local stability for $R_{0}<1$ and the endemic steady state is local stability for $R_{0}>1$.

\subsection{Numerical Solutions:}

Numerical method is used for solving numerical solutions of (9)-(13). We simulate our equations for disease free and endemic regions.

For disease free region:
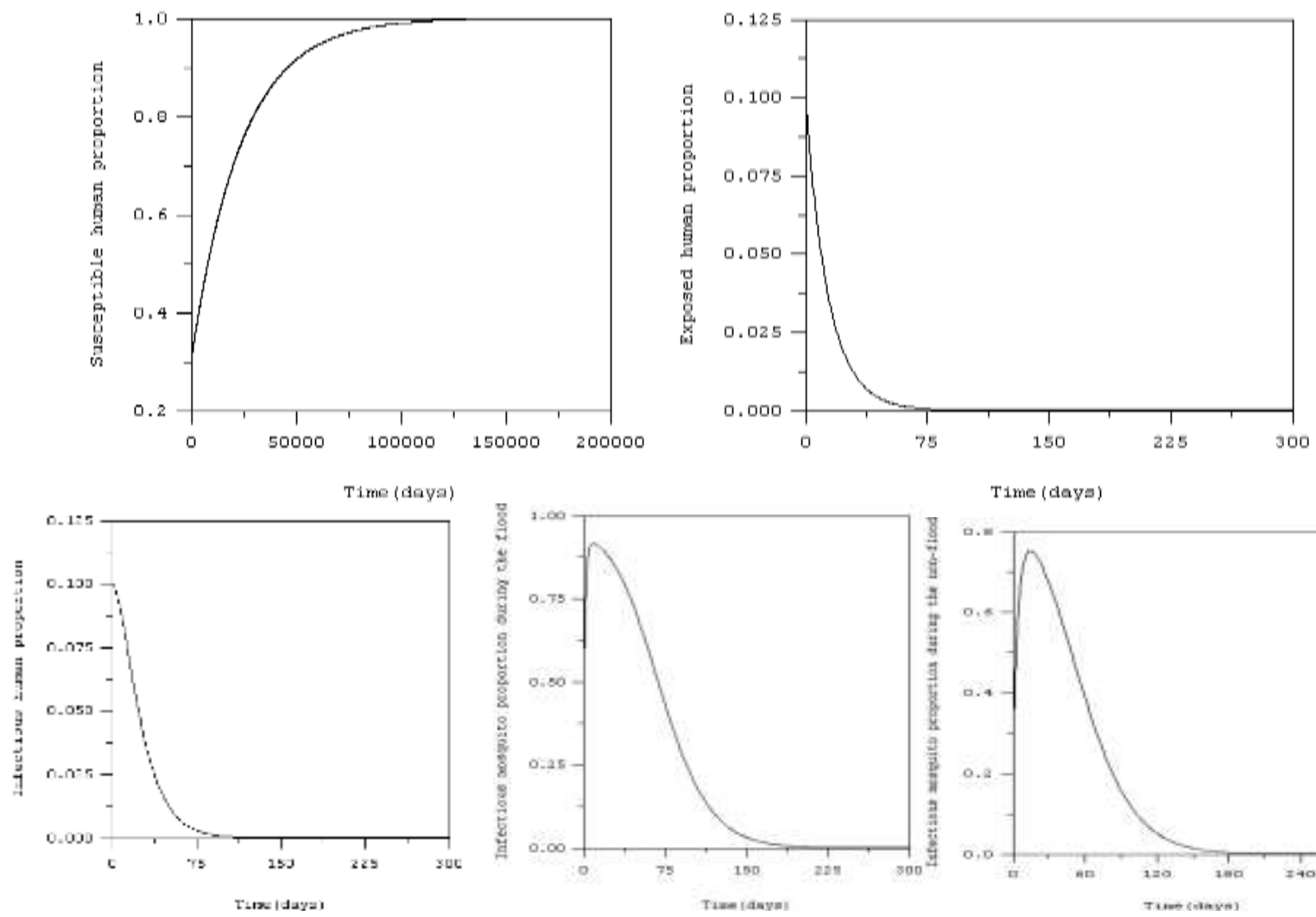

Time (daya)

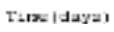

Trus Iany:

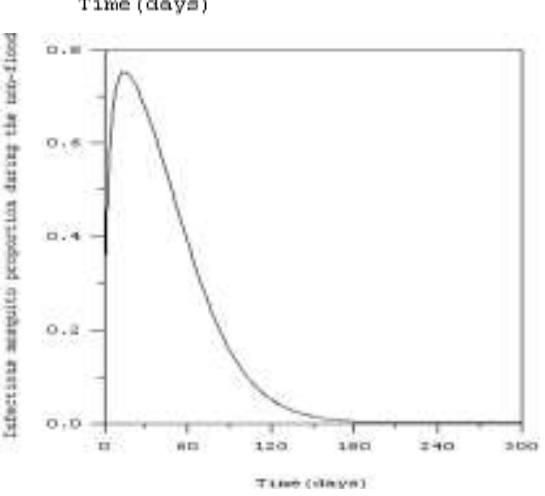

Fig. 1: Time series solutions of susceptible human, exposed human, infectious human, infectious mosquito during flooding time and infectious mosquito during non-flooding time on disease free region. The solutions converge to $(1,0,0,0,0)$

\section{For endemic region:}

The parameters are follows:

$\mathrm{h}=1 /(365 \times 65)$ corresponds to the life cycle 65 years of human. $\gamma_{\mathrm{n}}=0.006, \gamma_{\mathrm{v} f}=0.008, \gamma_{\mathrm{v}_{\mathrm{n}}}=0.002$ and $N_{\mathrm{h}}=10,000$ are arbitrary chosen parameters. $d_{v}=1 / 45$ corresponds to 45 days life time of Anopheles mosquitoes. $I I_{n}=14$ corresponds to 14 days of incubation period of Plasmodium malaria in human. $\gamma=1 / 14$ corresponds to the 14 days of recovering of human and $R_{0}=493$. 

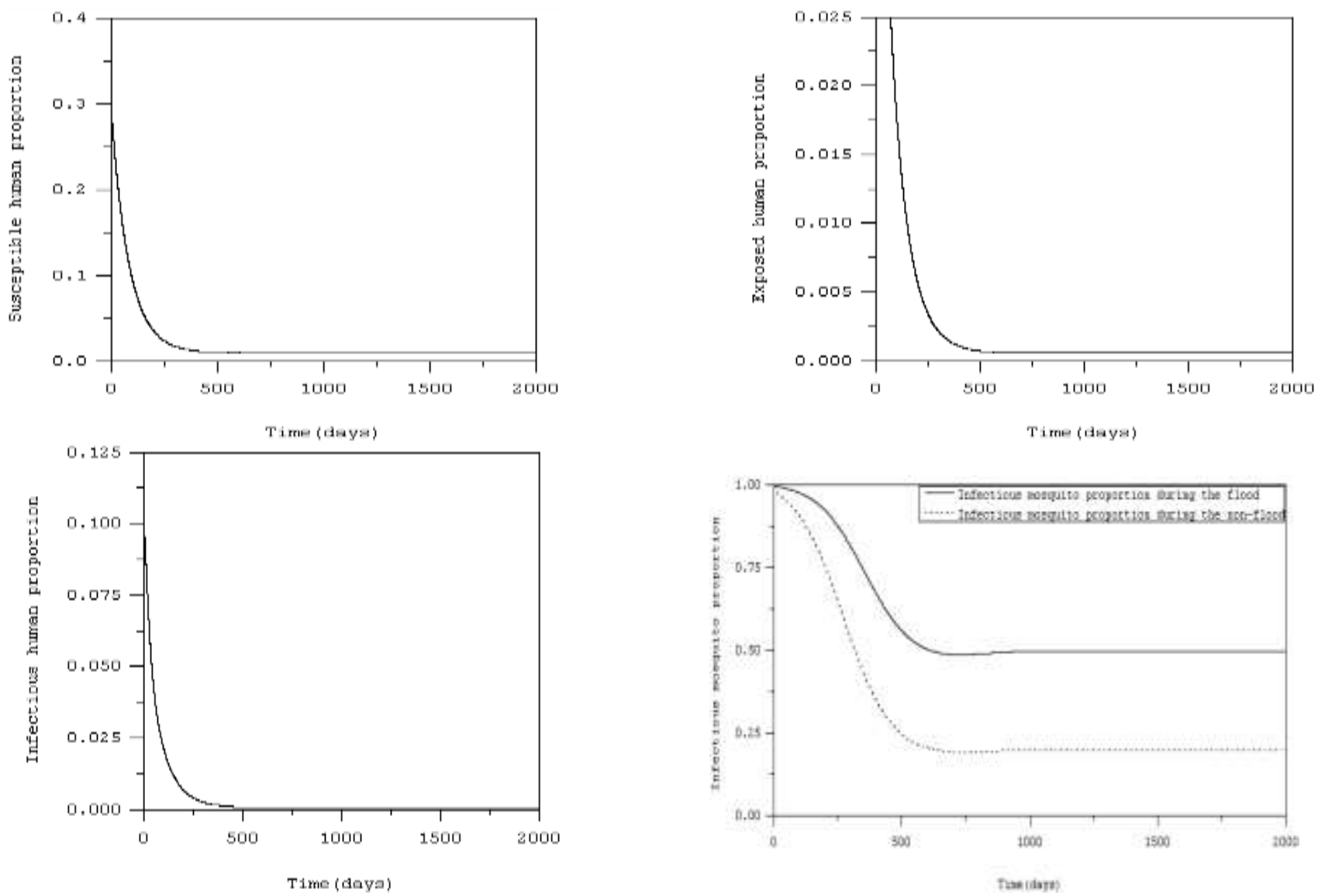

Fig. 2: Time series solutions of susceptible human, exposed human, infectious human, infectious mosquito during flooding time and infectious mosquito during non-flooding time on endemic region. The solutions converge to $(0.01,0.0006,0.0006,0.5,0.2)$

From the above figures, we can see that the solutions converge to the disease free steady state for $R_{0}<1$. For $R_{0}>1$, the solutions converge to the endemic steady state.

\section{Conclusions}

In this study, we formulate the model of Malaria transmission with the influence of flooding in Thailand. Condition for local stabilities of disease free steady state and endemic steady state is defined by $R_{0}$, where

$$
R_{0}=\frac{\gamma_{n} N_{n}\left(\gamma_{v_{f}}+\gamma_{v_{n f}}\right)}{d_{v}(d+\gamma)\left(1+d \mathrm{II}_{\mathrm{n}}\right)} \text {. }
$$

The basic reproductive number is given as $R_{0}^{\prime}=\sqrt{R_{0}}$, defined as the average number of secondary cases produced from primary cases [8]. From (15), we can see that the transmission rates of Plasmodium Malaria $\left(\gamma_{n}, \gamma_{v_{f}}\right.$ and $\left.\gamma_{v_{n}}\right)$ effect to the basic reproductive number. If we can reduce the transmission rate of this disease, then we can reduce the outbreak of the disease. Next, we simulate our solutions for different values of transmission rates. 

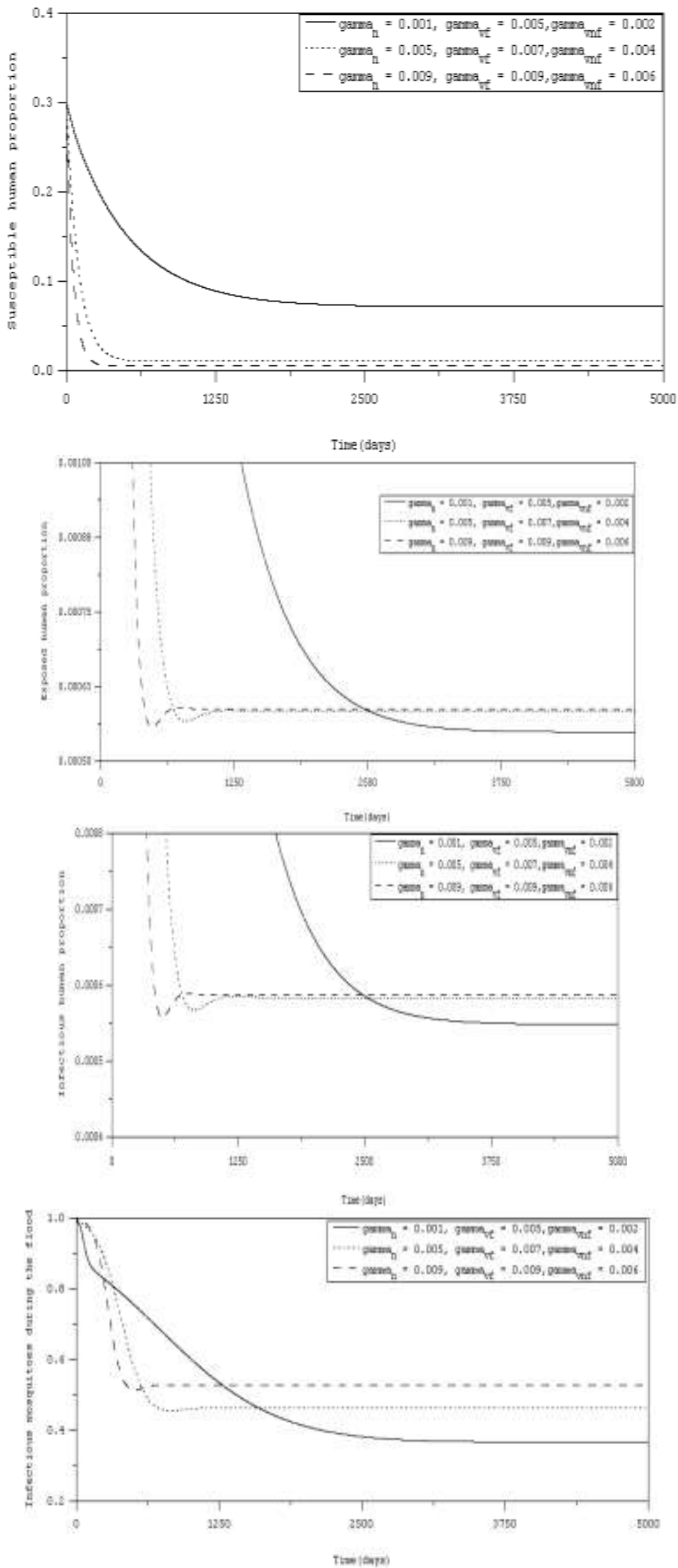


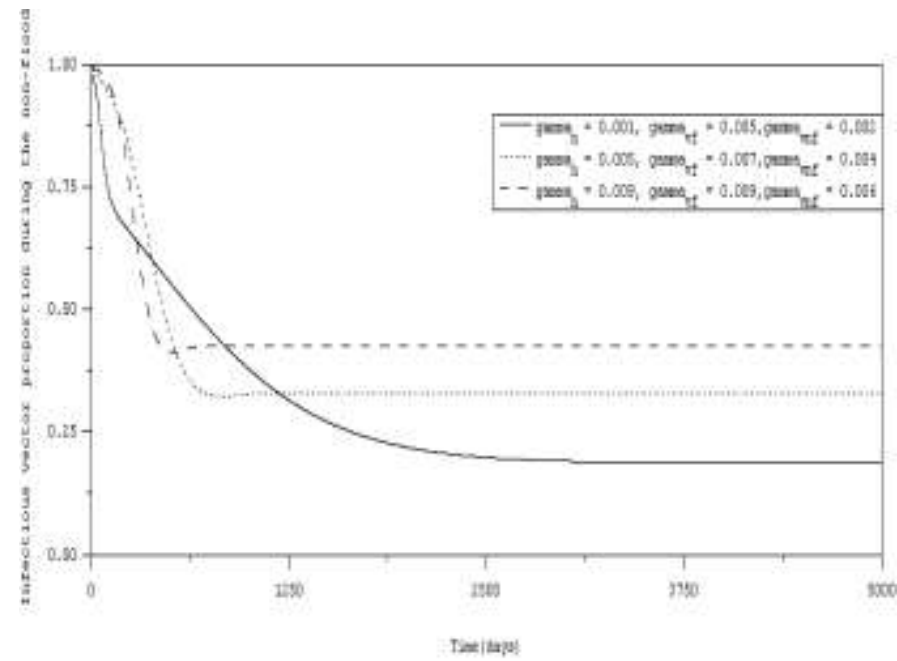

Fig.3: Time series solutions of susceptible human, exposed human, infectious human, infectious mosquito during flooding time and infectious mosquito during non-flooding time for the different sets of transmission rates.

We can see that when the transmission rates are higher, the steady state solutions of exposed and infectious groups are increasing but the steady state solutions of susceptible group is decreasing. From our simulations, we can see that the infectious proportion during flooding time is higher than the infectious proportion during nonflooding time. This is true because mosquitoes in the flooding time can grow faster than mosquitoes in the nonflooding time.

\section{Acknowledgements}

This work is supported by King Mongkut's Institute of Technology Ladkrabang and National Research Council of Thailand.

\section{References}

[1] T.H. Holtz, S.P.Kachur, J.R.MacArthur, J.M.Roberts, A.M.Barber, R.W.Steketee, et.al., Malaria surveillance. [online] Available fromhttp://www.cdc.gov/mmwr /preview/mmwrhtml/ss5005a1.htm.

[2] http://www.siamhealth.net/public_html/Disease/ infectious/malaria/ distribution.htm

[3] R.S.Phillipe, Malaria. London : Edward Arnold; 1983.

[4] J.P.Kreier, Malaria volume1 epidemiology, chemotherapy, morphology, and metobolism. New York:Academic Press; 1980.

[5] H.Mashaal, Clinical malariology : Southeast Asian Medical Information Center ; 1986.

[6] G.MacDonald, The epidemiology and control of malaria. London : Oxford University press ;1957

[7] A.Kammanee, N.Kanyamee, I.M. Tang, "Basic reproduction number for transmission of Plasmodium vivax malaria", Southeast Asian J Trop Med Publec Health 2001, 32, pp.702-6.

[8] L.Esteva, and C.Vargas, 1998. Analysis of a dengue disease transmission model. Mathematical Bioscience, 150, pp.131-151.

http://dx.doi.org/10.1016/S0025-5564(98)10003-2

[9] Leah EK., Mathematical models in biology, Random House, 1988.

[10] Hoffman JD., Numerical Methods for Engineers and Scientists, Singapore: McGraw-Hill. 